\title{
LAS EXPEDICIONES CIENTÍFICAS ESPAÑOLAS EN LOS SIGLOS XIX Y XX EN EL ARCHIVO DEL MUSEO NACIONAL DE CIENCIAS NATURALES
}

\author{
J. Luis Maldonado Polo \\ IH, CSIC, $\mathrm{H}^{\mathrm{a}}$ de la Ciencia
}

\section{RESUMEN}

Presentamos en este trabajo la catalogación de las series documentales sobre viajes y expediciones científicas españolas realizadas en los siglos XIX y XX, existentes en el Museo Nacional de Ciencias Naturales.

PALABRAS CLAVE: expediciones científicas, catálogo, siglos XIX y XX.

\section{SUMMARY}

This paper offers the catalogue of the documental series about spanish travels and scientific expeditions during the XIXth and XXth Centuries. These documents are at the Museo Nacional de Ciencias Naturales.

KEY WORDS: scientific expeditions, catalogue, $19^{\text {th }}$ and $20^{\text {th }}$ century.

El Museo de Ciencias Naturales de Madrid se fundó en 1771 bajo el reinado de Carlos III siendo nombrado director Pedro Franco Dávila quien aportó como donación al nuevo centro los especímenes y muestras de su gabinete privado formado en París durante su permanencia en esa ciudad francesa durante 14 años.

El Archivo con el trascurso del tiempo fue enriqueciendo sus colecciones con una gran cantidad de documentación manuscrita resultante de toda su actividad científica, pedagógica y administrativa.

El Museo de Ciencias Naturales posee una riquisima documentación, en gran parte conocida gracias a la labor de $\mathrm{M}^{\mathrm{a}}$ Ángeles Calatayud, archivera del centro, que con su trabajo a lo largo de los años ha podido sacar a la luz varios catálogos, editados por el CSIC, con información concerniente a los fondos del archivo del 
Museo y sobre viajes y expediciones científicas españolas a lo largo de los siglos XVIII y XIX ${ }^{1}$.

En estos catálogos se dan a conocer todos los pormenores que estos proyectos científicos generaron, quedando pendiente muchos otros fondos realtivos a otros viajes que tuvieron lugar con posterioridad y que nosotros hemos valorado y catalogado en una primera aproximación para que sirvan a los investigadores interesados en estos temas, poniéndo a su alcance una información desconocida que permitirá acrecentar los datos que este importante deposito documental contiene.

De manera que presentamos la selección, ordenación y catalogación de estas series documentales procedentes de las expediciones científicas realizadas por el estado español a lo largo de los siglos XIX y XX. La descripción de estos expedientes se ha realizado por orden cronológico, figurando en cada ficha un número de orden correlativo, con un total de 247 registros catalográficos.

La documentación la hemos clasificado en los siguientes apartados²:

1.- Exploraciones científicas al Africa Occidental. 1886-1915

2.- Comisión permanente para la exploración y estudio del noroeste de Africa. 1901-1915

3.- Expedición a Canarias de Cesar Labrado. 1905-1906

4.- Viaje al Golfo de Napoles. [1905]

5.- Comisión Científica a Galicia. 1921-1929

6.- Estudios y envíos de materiales zoológicos de Guinea. 1924-26

7.- [Excursión del Sr. Lozano a Río de Oro]. 1933

8.- Estudios y exploración florística de la Mancha de G. Albó. 1934

9.- Proyecto de Expedición Transafricana. 1933-36

10.- Comisión Científica de Ifni. 1834-35

11.- Expedición del capitán Iglesias al Amazonas. 1936

12.- Campañas Ictiológicas y pesqueras. 1939

13.- Trabajos preparatorios de la expedición a Guinea e Ifni. 1939-1940

14.- Expedición a Filipinas. S.F.

1 Calatayud, M. a A. (1984), Catálogo de las Expediciones y Viajes Científicos Españoles, siglos XVIII y XIX, Madrid, CSIC. Calatayud, M. ${ }^{a}$ A. (1987), Catálogo de Documentos del Real Gabinete de Historia Natural (1752-1786), Madrid, CSIC-MNCN.

2 Los apartados independientes en que se ha divido la documentación, considerando los viajes por separado, lo hemos hecho de esta forma por razones de clasificación al encontrarse la información incluída en expedientes individuales. Advertimos, por tanto, que existe en algunos casos verdadera relación entre unos y otros solapándose los documentos de algunos grupos que hacen referencia al mismo viaje o proyecto, al existir vínculos científicos con las zonas o países a los que nos referimos desde tiempo atrás. Se realizaron viajes y estrategias político-científicas a lo largo de los años con clara relación a los antecedentes existentes, confecionándose proyectos nuevos o continuando los ya comenzados o simplemente sugeridos. Esta actividad generó en los años sucesivos alguna documentación que figura por este motivo en expedientaes separados. 


\section{CATÁLOGO DE LOS FONDOS DOCUMENTALES DE LAS EXPEDICIONES CIENTÍFICAS DE LOS SIGLOS XIX Y XX.}

\section{EXPLORACIONES CIENTÍFICAS AL ÁFRICA OCCIDENTAL. 1886-1915.}

$1 \quad$ 1886, febrero 20, Madrid

Relación de objetos necesarios para la exploración científica en el Africa Occidental. Por Francisco Quiroga. 3 h.

Comunicación al Director del Museo de Ciencias Naturales que se ha acordado incorporar a la expedición, al ayudante del Museo y profesor de la Facultad de Ciencias de la Universidad Central, Francisco Quiroga. 2 h. Corre unido: Oficio de Francisco Quiroga de 6 de abril tomando posesión de ese cargo. $2 \mathrm{~h}$.

Oficio del Rector de la Universidad Central al Director del Museo de Ciencias Naturales en el que se le comunica la decisión de la reina de que Quiroga cobre el sueldo y gratificaciones correspondientes durante la Expedición 2 h. Corre unida: Notificación de 20-IV, del Director del Museo al ordenador de pagos del Museo de Fomento de esta decisión. $1 \mathrm{~h}$.

Carta al Director General de Instrucción Pública acerca de que Sorela y Montes de Oca recojan objetos naturales para el Museo por lo que necesitan instrumentos adecuados para ello, por lo que se pide tres o 4000 rs. 1 h. Corren unidos: Comunicación de 5 de agosto al Director General de Instrucción Pública, sobre la insuficiencia de las 1.000 pesetas concedidas para que la Expedición se prolongue más tiempo del previsto. $1 \mathrm{~h}$. Comunicación duplicada de 6 de agosto de Miguel Colmeiro al Director del Museo, trasmitiéndole la comunicación de haberse concedido 1.000 pts para adquisición de instrumentos para las expediciones de Africa. 4 h. Otra de octubre 2, Madrid. Oficio al Rector de la Universidad Central remitiéndole las cuentas del Museo y sobre las 2.000 pts concedidas para la adquisición de instrumentos. $1 \mathrm{~h}$.

$5 \quad$ 1886, septiembre 8, $\mathrm{St}^{\mathrm{a}}$ Cruz de Tenerife.

Carta de Francisco Quiroga a Miguel Maisterra sobre el envío desde Canarias del material que había sacado del Museo y los objetos recolectados en Africa y en Canarias. 2 h. Corre unida: Comunicación de 15 de septiembre de José Verdugo sobre este envío. 1 h.

$6 \quad$ 1886, septiembre 18, Madrid.

Comunicación de F. Quiroga al Director del Museo expresando su regreso de la Expedición a Africa. 2 h. Corre unido: Oficio de 15-XI al Ordenador de pagos del Museo de Fomento comentando esta circunstancia. $1 \mathrm{~h}$. 
1886, octubre 2, Madrid.

Cuentas. Véase $\mathrm{n}^{\circ} 4$.

1886, [noviembre, Madrid].

Oficio al ministro de Fomento comunicándole el acuerdo de la Junta de Profesores del Museo de Ciencias del 8 de ese mes, sobre las recolecciones y descubrimientos científicos realizados por los expedicionarios y en especial por Francisco Quiroga. $1 \mathrm{~h}$.

$9 \quad[1886]$

«Fragmentos integrantes al anterior Opúsculo». Es una descripción geográficonaturalista de las islas Canarias... 4 h., f.

II. COMISIÓN PERMANENTE PARA LA EXPLORACIÓN Y ESTUDIO DEL NOROESTE DE ÁFRICA. 1901-1915.

10 1901. noviembre 25, Madrid.

Carta del Duque de Almodovar a Ignacio Bolívar transcribiéndole el nombramiento de Presidente de la Comisión encargada del estudio de los recursos naturales de las posesiones españolas de Africa Occidental según la R.O de esta misma fecha en que se creó esta Comisión. 2 h., f.

11 [1901].

Carta del Subsecretario del Museo de Estado, José Pérez Caballero a Ignacio Bolívar, transmitiéndole la R.O de esa fecha en la que figura la composición de la Comisión. Corre unido: Carta de 26 de diciembre, Madrid, sobre la confirmación de la exploración para Museos y el cambio de artículos del presupuesto colonial. $1 \mathrm{~h}$.

12 1902, enero 9, Madrid.

Recorte del «Imperial», incluyendo una noticia de la Sociedad Geográfica con la conferencia de Manuel. Martínez de la Escalera.

13 1902, febrero 20, Madrid.

Carta José Pérez Caballero a Ignacio Bolívar diciéndole que el Gobernador General de Fernando Poó remite al $\mathrm{M}^{\mathrm{o}}$ muestras de productos naturales de esa colonia.

14 1902, marzo 10, Madrid.

Carta del Marqués de Almodovar al Director del Museo de Ciencias Naturales (I. Bolívar) diciéndole que ha recibido su comunicación de 5 del corriente. Se contesta en base a la propuesta hecha para Ángel Cabrera, encargado de las colecciones de mamíferos de la Comisión, aceptado la nominación de «Herpestes Almodovari» de la especie nueva encontrado para Escalera en Río Muni.

15 1902, mayo 28, Madrid.

Comunicación de Blas Lázaro Ibiza a [Bolívar] remitiéndole un informe sobre la «naturaleza y aplicaciones» de productos naturales del Golfo de Guinea. Se incluye el citado informe. 


\section{LAS EXPEDICIONES CIENTÍFICAS ESPAÑOLAS EN LOS SIGLOS XIX Y XX}

1902, junio 10, Madrid.

Carta a José Pérez Caballero sobre que Blas Lázaro Ibiza informe de los productos de Fernando Poó. (Es borrador).

1902, septiembre 2, Madrid.

Carta del Subsecretario del Ministerio de Estado a Ignacio Bolívar dándose por enterado del informe de Blas Lázaro Ibiza, acordándose se publique y pidiéndole la devolución de las muestras para exponerlas en el Ministerio.

1902, septiembre 9, Madrid.

Comunicación del Subsecretario del Ministerio de Estado, Gutiérrez y Ossa a Ignacio Bolívar que han recibido las citadas muestras de Río Muni.

19 1902, septiembre 17, Madrid.

Contestación de Ignacio Bolívar a Gutiérrez y Ossa sobre el traslado de las muestras de minerales que le había remitido para que las examine Salvador Calderón.

20 1902, septiembre 29, Madrid.

Carta de José Pérez Caballero a Ignacio Bolívar comunicándole que informe sobre la preparación de las colecciones recogidas por Manuel Martínez de la Escalera y que el Ministro acceda a la petición de publicación de un libro con los resultados. De igual manera se acepte la aportación de 4.000 pts para ello, pero este donativo se hará en el ejercicio del año siguiente.

21 [Sin fecha].

Informe de la Comisión sobre las gestiones para el estudio por especialistas de las colecciones africanas de Manuel Martínez de la Escalera. Se incluye los especialistas tanto españoles como extranjeros. $3 \mathrm{~h}$.

Comunicación del Jefe de la Sección Colonias del Ministerio de Estado a Ignacio Bolívar sobre hacer efectivo el cobro pendiente. $1 \mathrm{~h}$.

Oficio de Rodíguez San Pedro a Ignacio Bolívar sobre que al rey ha accedido a su solicitud de 14-VI-1902 y se le facilite la suma de 4.000 pts. Nota sobre presupuestos de los viajes y pertrechos de los expedicionarios. $1 \mathrm{~h}$.

1904, febrero 7, Madrid.

Carta de gratitud de la Comisión y de la Real Sociedad Española de Historia Natural (RSEHN), para la ayuda sobre la publicación del tomo $1^{\circ}$ de las memorias de dicha Sociedad dedicado a la fauna de África Occidental. Sobre las colecciones de Escalera que no son suficientemente completas para su publicación. 
1904, febrero 26, Madrid.

Comunicación a Ignacio Bolívar de que el Ministro de Estado queda enterado del contenido de la comunicación del 7 del corriente, referente a la publicación de las Memorias sobre la Fauna.

Comunicación de E. de Ojeda a Ignacio Bolívar sobre la inversión de 1.559 pts y 66 cmos de los 4.000 concedidos para la publicación de los 2 Boletines de la R.S.E.H.N.

Eduardo Bosch remite a Ignacio Bolívar una comunicación que por error había sido enviada a Fernando Poó.

Borrador sobre la propuesta al ministro de los gastos de la Comisión. 1 h. Corre unido: «Enmienda al presupuesto de las posesiones de Africa». $1 \mathrm{~h}$.

29 1905, febrero 20, Tánger.

«Expedición Comercial al interior de Marruecos». Fecha de salida, itinerarios, pertrechos, etc. $3 \mathrm{~h}$.

301905 , marzo 9, Santiago.

Carta de César Sobrado a Martínez de la Escalera comentándole que está dispuesto para el viaje hasta Madrid, para emprender la excursión científica que se tiene prevista. 2 h.

31 1905, marzo 29, Madrid.

Carta de W. Weller a Vicente Vera ofreciendo sus servicios como intérprete para la Expedición. 2 h.

32 1905, abril 3, Casablanca.

Carta a Bolívar anunciándole que le envía insectos y demás objetos naturales y prestando su ayuda para facilitarle excursión que tiene previsto realizar a esos territorios. $2 \mathrm{~h}$.

33 1905, abril.

Excursión a Ceuta del Dr. Lucas Fernández Navarro. Facturas de hoteles en Málaga, Algeciras y Gibraltar y resumen de los gastos de la excursión, fechado en Madrid el día 26. 4 h. Corren unidos: Notas de gastos de la excursión en los meses de junio, julio y agosto en Málaga, Peñón de Vélez, Alhucema, Ronda y Mélilla. 12 h. Recibo de Lucas F. Navarro de 2.500 pts., por gastos de su próxima excursión por el N. de Africa. $1 \mathrm{~h}$.

34 1905, abril 19, Algeciras.

Carta de Lucas Navarro a Ignacio Bolívar comentándole que pasará a Ceuta donde recogerá productos naturales. $2 \mathrm{~h}$. 


\section{LAS EXPEDICIONES CIENTÍFICAS ESPAÑOLAS EN LOS SIGLOS XIX Y XX}

1905, abril 26, Casablanca.

Carta de Adriano a Ignacio Bolívar recomendándole algunas precauciones para su proyectada excursión por Marruecos. $2 \mathrm{~h}$.

Comunicación de Manuel Allende Salazar al Duque de Medinaceli y Manuel Martínez de la Escalera anunciándole que la Comisión se reunirá a $1^{\circ}$ de mayo a las 12 h, en el Museo. 4 h. Corre unido el Libro de Actas de la «Comisión permanente para la exploración del Noroeste de Africa de la Real Sociedad Española de Historia Natural». Contiene:

- Sesión de Constitución de 1 de mayo de 1905

- Relación de pertrechos y material de campaña

- Relación de nóminas del personal integrante

Son 13 páginas +177 en blanco

1905, mayo 1, Madrid.

Boletín de suscripción de la RSEHN para sufragar gastos de la exp. al NO. de Africa. 2 h., f., mec.

38 1905, mayo 12, Santiago.

Carta de Cesar Sobrado a Ignacio Bolívar sobre su aceptación para formar parte en la proyectada excursión para el NO. de Africa para lo cual podría embarcarse en algún puerto de Galicia con destino a Canarias y de éstas a Marruecos. 2 h. Véase p. 14.

39 1905, mayo 16.

Carta a Emilio Ribera sobre una nota de Escalera para el rey quién recomendará al Ministro de Estado su contenido. 2 h. Véase lo siguiente: 1905, marzo 30 - junio 12. Serie de notas y saludos sobre la visita al rey y los trámites para ello. $17 \mathrm{~h}$.

$40 \quad 1905$, mayo 17.

Comunicación a Ignacio Bolivar sobre las 5.000 pts. concedidas por el rey para la expedición de África. $1 \mathrm{~h}$. Corre unido: carta al Marqués de Urquijo de 11 de mayo de 1905 comentando esta circunstancia y el envío de el «Libro talonario» y otros documentos útiles. $1 \mathrm{~h}$.

$41 \quad 1905$, mayo 18.

Carta de Manuel Allende Salazar al Marqués de $\mathrm{St}^{\mathrm{a}}$ Cruz diciéndole que no puede acompañar a la Comisión para el estudio del NO. de Africa, a la visita al rey por lo que le pide que sea él quién presida la visita. $2 \mathrm{~h}$. Acompañan: Salutación del margen de $\mathrm{St}^{\mathrm{a}}$ Cruz a Ignacio Bolívar sobre el mismo asunto de 22 de mayo. 2 h. Otra de 25 de mayo sobre la audiencia a los monarcas. $2 \mathrm{~h}$.

42 1905, mayo 22, Madrid.

Factura de Agustín Fernández (grabador de metales), de un sello de caucho con la inscripción «Comisión permanente para la exploración del Noroeste de Africa». 1 h. 
43 1905, mayo 26.

Carta de Anselmo González a Ignacio Bolívar sobre incluir noticias y retratos de los expedicionarios en varios periódicos. $2 \mathrm{~h}$. Corre unido: Borrador de 25 de mayo sobre la indecisión de noticias de la exploración de Marruecos. 2 h.

$44 \quad 1905$, mayo 27.

Carta de Allende Salazar sobre su imposibilidad de conocer la suma con que contribuirán los Vicepresidentes de la Comisión de Marruecos, él por su parte lo hará con 500 pts. $2 \mathrm{~h}$.

1905, mayo 31, Cartagena.

Notificación del Diputado para esa ciudad a Ignacio Bolívar lamentando que el Diario Universal haya incluido un artículo titulado «Marruecos». $2 \mathrm{~h}$. Corre unida nota a Ignacio Bolívar sobre su ayuda para indicar al Director del «Diario» las observaciones que desea. $2 \mathrm{~h}$.

46 1905, junio 12, Madrid.

Carta del Conde de Mallada a Emilio Ribera, sobre las ayudas económicas y de instrumentos que necesitan los comisionados para Marruecos. $1 \mathrm{~h}$.

47 1905, junio 25.

Carta del Conde de Mallada a Emilio Rivera sobre que la Infanta Eulalia le ordenó comprar los primarios «Goerz» que le remite. $2 \mathrm{~h}$.

48 1905, julio 14, San Ildefonso

Notificación de Alonso Coello de remisión de 1 cheque de 500 pts., al marqués de $\mathrm{St}^{\mathrm{a}}$ Cruz. $2 \mathrm{~h}$.

49 1905, agosto 24, Guernica.

Comunicación de Manuel Allende Salazar a I. Bolívar sobre el Real Decreto referente al Laboratorio de Biología de Mogador. 1 h. Corren unidos: Otra del 15 de noviembre sobre el presupuesto del Laboratorio. Otro borrador de 20 de noviembre dirigido al Ministro de Estado para que se gestione la cesión del edificio en ruinas «Palacio del sultán» en Mogador para instalar el laboratorio. $1 \mathrm{~h}$. Nota sobre este asunto. $1 \mathrm{~h}$. Otra sin fecha sobre lo mismo. $1 \mathrm{~h}$. Otra del 14 de agosto, desde Guernica. $1 \mathrm{~h}$.

$50 \quad 1905$, noviembre 15, Madrid.

Borrador de notificación al Marqués de $\mathrm{St}^{\mathrm{a}}$ Cruz nombrándole presidente en la Comisión en asuntos económicos junto a Luis Bahía y Manuel Antón. 1 h.

$51 \quad 1905,18,20,22$, de noviembre.

Sobre el edificio de Mogador. Véase, $n^{\circ} 49$. 


\section{LAS EXPEDICIONES CIENTÍFICAS ESPAÑOLAS EN LOS SIGLOS XIX Y XX}

52 1905, noviembre 27, Orense.

Certificado de Antonio Martínez Rico sobre el acuerdo adoptado el 20 de julio referente a la imposibilidad, por falta de fondos de poder contribuir a las fotos de la Comisión al NO. de Africa. 2 h., f.

53 1905, noviembre 28, Madrid

Oficio del Subsecretario de la Sección $3^{\mathrm{a}}$ de Bellas Artes al Presidente de la RSEHN sobre la denegación del material de campaña y armamento solicitado por la Sociedad, para los trabajos en el NO. de Africa. $2 \mathrm{~h}$.

54 Borrador [sin fecha], referente a la necesidad de extender los recursos naturales para el NO. de Africa y propuesta de inclusión de 60.000 pts como presupuesto... 2 h.

$55 \quad 1905$, diciembre 1.

Sobre presupuesto del laboratorio de Mogador. Véase n ${ }^{\circ} 49$.

56 1905, diciembre 29, Madrid.

Comunicación del Diputado por Cartagena a Ignacio Bolívar sobre que apoyará su iniciativa cuando se trate en la Censura de las preguntas coloniales. $2 \mathrm{~h}$.

57 1905, diciembre 31, Valladolid

Carta de José del Muro a Ignacio Bolívar mostrando su discrepancia sobre las cifras de gastos de la Sociedad Española de Historia Naural y de los destinados a la exploración del NO. de África. $2 \mathrm{~h}$.

$58 \quad 1906$, enero 7

Sobre plazas vacantes. Véase $n^{\circ} 49$

59 1906, enero 16.

Carta de José $\mathrm{M}^{\mathrm{a}}$ Dusmet a Ignacio Bolívar sobre la convocatoria de una reunión para tratar de las exploraciones que sean convenientes a la Sociedad. $2 \mathrm{~h}$.

60 1906, mayo 27. Madrid.

Carta de Luis Bahía al Presidente de la Comisión Permanente y de la RSEHN. disculpando su asistencia a la Junta convocada para el día siguiente. $1 \mathrm{~h}$.

61 1906, noviembre 6, Tánger.

Carta de Manuel de la Escalera [a Bolívar] sobre la posibilidad de conseguir el Palacio del Sultán. Anucia que enviará mil ortópteros y comenta noticias de otros insectos con sus costes que ha pagado él mismo... $4 \mathrm{~h}$. Corren unidos: Recibo de Vancher de una cantidad dada para M. de la Escalera. Anotaciones al margen de 9 de noviembre del mismo Escalera sobre el envío desde Tánger de 2 cajas para la RSHN. 1 h. Recibo con fecha 14 de noviembre de Riggmbar desde Mogador sobre una cantidad dada por Escalera. Atención al margen sobre entrega de 1 caja al Consulado a Mogador. 1 h. [1906]. Anotaciones de cuentas con cantidades recaudadas. $2 \mathrm{~h}$ 
62 [1906, noviembre] 7, Tánger.

Carta de Martínez de la Escalera [a Bolívar] sobre la entrega a la Agencia de la Trasantlántica de las 2 cajas con ortópteros y con los especímenes en alcohol y noticia sobre otras recolecciones. También le habla sobre su viaje al sur... $2 \mathrm{~h}$.

63 1906, noviembre 11. Mogador.

Carta de Martínez de la Escalera [a Bolívar] contándole pormenores de su llegada a esta población y de que Riggmbach sale para Tumbuctú y le ha dado más de 150 mamíferos y sobre el costo de estas. También comenta noticias de la compra del rifle Winchester y una escopeta de caza. Igualmente le dá noticias de que partirá para cabo Luby. $2 \mathrm{~h}$.

64 [1906] Algeciras.

Carta de Martínez de la Escalera [a Ignacio Bolívar] sobre sus recolecciones en esta zona y la imposibilidad de embarcarse por motivos del temporal que asola el Estrecho... $2 \mathrm{~h}$.

65 1906, diciembre 1, Mogador.

Carta de Martínez de la Escalera a Ignacio Bolívar comentándole que llegó el día anterior de Cabo Luby con los consiguientes inconvenientes y pormenores en el puerto y en el barco. «Cartagena». Se comenta que Abdelkrim es el interprete del barco y que ha traído 27 ratas y otras especies faunísticas. Comenta detalles del viaje... $2 \mathrm{~h}$.

66 1906, diciembre 30, Mogador.

Carta de Martínez de la Escalera [a Bolívar] diciéndole que carece de sus noticias y desconoce si ha recibido los 2 envíos de Tánger y de Mogador, así mismo le comenta que prepara un tercer envío especificando los pormenores de la remesa. También le informa que piensa partir para Marraquech y que ha escrito a Allende Salazar contándole la situación política. $2 \mathrm{~h}$.

67 1907, enero 10, Mogador.

Carta de Martínez de la Escalera [a Bolívar] comentándole que al día siguiente sale para Marraquech, también que enviará en el vapor «Cartagena» tres cajones de productos naturales... $2 \mathrm{~h}$.

68 1907, enero 11, Mogador.

Carta de Martínez de la Escalera a Emilio J. Navarro sobre la autorización para desembarcar las armas. $2 \mathrm{~h}$.

69 1907, enero 15, febrero y abril 20, Madrid.

Acuerdos de la R.S.E.H.N. sobre la continuación de los trabajos de exploración en Marruecos. El Comisario de la Sociedad continuará en Mogador y desde aquí se trasladará a Marraquech donde explorará el territorio del Sur y el Atlas. Igualmente se acordó formar una Comisión para el estudio geológico de las islas Canarias y encargarse de la Presidencia de la Comisión permanente de la Soc. José Muro así como de la llegada de Escalera a la capital de Marraquech. $3 \mathrm{~h}$. 


\section{LAS EXPEDICIONES CIENTÍFICAS ESPAÑOLAS EN LOS SIGLOS XIX Y XX}

70 1907, enero 22, Marraquech.

Carta de Martínez de la Escalera [a Bolívar] comentándole que lleva ya 4 días en esta localidad..., que Pepe sale todos los días al campo a coger coleópteros. El por su parte compra en el mercado otros productos de interés naturalista. Comenta circunstancia del viaje desde Mogador y del previsto a partir de esos momentos. $4 \mathrm{~h}$. Corre unido: Borrador sobre estos pormenores. $1 \mathrm{~h}$.

71 1907, febrero 19, Marraquech.

Carta de Martínez de la Escalera [a Bolívar] que está a punto de salir para Madrid donde tiene previsto llegar hacia el 10 de marzo, acompañado de un moro de buena posición y con el que hará viajes por la Península. $2 \mathrm{~h}$.

72 1907, marzo 8, Barcelona.

Carta de Odón de Buen a Ignacio Bolívar, sobre que ha encontrado las cajas de Mogador y que se expedirán a Madrid. $2 \mathrm{~h}$.

73 1907, abril 2 y 4, Madrid.

Carta de Martínez de la Escalera [a Bolívar] sobre trámites al margen de la Expedición. 2 h.

74 1907, abril 22, Madrid.

Carta de Lucas Navarro a Ignacio Bolívar adjuntándole una carta. 1 h. Se incluye la carta dirigida al Presidente de la Comisión para la Exploración del NO. de Africa. En ella comenta los trabajos realizados en sus exploraciones geológicas y que tiene entregado a la RSEHN una Memoria sobre «Observación Geológicas de la Isla de Hierro» y tiene otra sobre Africa. Resalta la importancia de estudiar la Islas Canarias. También incluye una nota sobre los costos de su viaje. $2 \mathrm{~h}$. Corre unido: un «Plan probable de excursión geológica», duración, itinerario, presupuestos y mapa del NO. de Africa. $1 \mathrm{~h}$.

75 1907, mayo 12, Casablanca.

Carta a Ignacio Bolívar contando el paso de Martínez de la Escalera por Mogador y Marraquech. $1 \mathrm{~h}$.

$76 \quad$ 1907, mayo 15.

Carta de Martínez de la Escalera [a Bolívar] que queda en Marraquech, su compañero Pepe y él vuelve a embarcarse para volver a Safli e incorporarse al nuevo Gobernador de Marraquech. También comenta algo sobre el destino del cajón con objetos en alcohol que salió de Marraquech. $2 \mathrm{~h}$.

77 1907, junio 5, Mogador.

Carta de Martínez de la Escalera [a Bolívar] recomendando a Antonio Benitez y Ortiz. También que va a emprender la ruta hacia el Norte, mientras Pepe está en Mazayan y Hanut en Marraquech. 2 h. 
78 1907, junio 12, Mogador.

Carta de Martínez de la Escalera [a Bolívar] comentándole su desesperación por no poder ir al Atlas y el destino que da a los otros colaboradores. También le informa de la situación política. $2 \mathrm{~h}$.

79 1907, diciembre 10 y 25, Madrid.

Dos salutaciones del Ministro de Estado a Ignacio Bolívar, informándole sobre su recibimiento de los ejemplos de la Sociedad y de las cuentas de la sbvención del Ministerio para la «Comisión de Muni». $4 \mathrm{~h}$.

80 [1907].

El presidente de la Comisión Permanente de la SEHM al ministro sobre el estudio del Río Muni, remite los números del Boletín de los años 1906 y 1907 en los que aparecen cuentas para la publicación de un tomo de Memoria sobre la fauna de Guinea Española. $1 \mathrm{~h}$.

81 [1908].

Informe (borrador) sobre decisiones referente a la Exploración del NO. de Africa. Creadas por Real Decreto 24-VIII-1805, la Estación de Biología marina en Mogador, presupuesto de gastos de 1806 y 1807 y sobre el viaje del ayudante de la estación José Taboada por Europa. $3 \mathrm{~h}$.

82 [1908].

Relación de cuentas y gastos en relación a la exploración del NO. de Africa. 2 h., f.

83 1908, febrero 3 París (en francés)

Sobre unos huevos de coleópteros... de recolecciones de Martínez de la Escalera. 2 h.

84 [Sin fecha].

Comunicación [a Ignacio Bolívar] sobre diferentes cuestiones relacionadas con los antecedentes de la gestión de apoyo del Ministerio de Estado para el estudio de Africa. $1 \mathrm{~h}$.

85 1911, noviembre, Madrid.

Carta sobre la R.O. del Ministerio de Estado de octubre sobre remisión de ejemplares de himenópteros y otros al Museo así como la publicación de una memoria, sobre la publicación de los «coleópteros de Marruecos y la pensión que necesita para estudiar en París y Londres, solicitando autorización para ausentarse temporalmente de Marruecos. $1 \mathrm{~h}$.

86 [1911].

Notas sobre fauna y geología del NO. de Africa y Canarias.

87 1913, marzo 16, Madrid.

Acuerdo de la Junta Directiva de la R.S.E.H.N. sobre el nombramiento de vocales de la Comisión Permanente para la exploración del NO. de África. 1 h. (mec). 


\section{LAS EXPEDICIONES CIENTÍFICAS ESPAÑOLAS EN LOS SIGLOS XIX Y XX}

1913, marzo 18. Madrid.

Saludo del Marqués de Urquijo a Ignacio Bolívar sobre el saldo de 5.532 pts a favor de la RSEHN. $1 \mathrm{~h}$.

89 1913, marzo 23. Tánger.

Informe de Manuel Martínez de la Escalera sobre el proyecto de exploración del NO. de África. Comenta diversos asuntos de su actividad como la de otros comisionados y la creación en Tánger de un «Laboratorio-Hospedería de Biología». 2 h. (mec).

1913, marzo 25, Madrid.

Comunicación del acuerdo de la Comisión del NO. de Africa de la R.S.E.H.N. sobre la reanudación de los trabajos de exploración y estudios de los territorios de Marruecos. 1 h.

91 1913, marzo 28, Madrid.

Carta al Ministro de Estado comunicándole la decisión tomada por la Comisión del NO. de África sobre la recomendación de los trabajos de exploración y estudio de territorio de Marruecos, creándose una nueva expedición de naturalistas. 2 h., f., (mec).

1913, marzo 28, Madrid.

Comunicación a Ignacio Bolívar del Decano de la Facultad de Ciencias de la Universidad Central, disculpando su asistencia a la Junta de la Comisión. 3 h.

1914, marzo 4, Madrid.

Contestación a Ignacio Bolívar sobre 2 cartas de pago. 1 h.

94 1914, diciembre 27. Tetuán.

Comunicación a Ignacio Bolívar sobre los 100 ejemplos que ofrece de la obra «Yebala y el bajo Luccus» la Sociedad. $2 \mathrm{~h}$. Corren unidos: Carta del Comandante General de Melilla de 25 de enero de 1915 a Ignacio Bolívar, sobre el recibo de una carta de 31 de diciembre y de los 100 ejemplares del libro «Yebalo...» 2 h. Otra de Roberto Cano del 13 de abril de 1915 desde Melilla agradeciendo la remisión del mismo libro. 2 h. Otra del Comandante General de Lurache de 3 de febrero agradeciendo el envío de 50 ejemplares más de ese libro. 1 h. (mec). Otra de Pablo Vallescá de 12 de abril en agradecimiento del envío de 1 ejemplar de esa obra. 2 h. Otra de Roberto Cano de 12 de abril fechada en Melilla sobre un ejemplar recibido de ese libro. 2 h. Otra del Presidente General de la Junta de Fomento de Melilla al Presidente de la R.S.E.H.N. Fernando García Arenal sobre haber recibido 1 ejemplar. 1 h. (mec). Obra de José $\mathrm{M}^{\mathrm{a}}$ Paniagua a F. García Arenal agradeciéndole el ejemplar enviado. 2 h. Otra fechada en Madrid el 21 de diciembre de 1914, remitiéndo 5 ejemplares del libro. $1 \mathrm{~h}$.

95 1915, enero 4, Madrid.

Carta de los Comisionados por la RSEHN para el examen de sus cuentas anuales y de los de la expedición a Marruecos, verificada en abril-junio de 1913 y sobre la publicación del libro «Yebala...» 1 h., f. (mec). 


\section{J. LUIS MALDONADO POLO}

96 [1915].

Carta al Gerente de una editorial de Barcelona mostrando su desacuerdo por haberse incluido en el libro publicado para Casa «Una expedición Científica a Marruecos», grabados y texto que forman parte de la otra obra. «Yebala y el bajo Luccus». $1 \mathrm{~h}$.

97 diciembre 28 [?].

Carta a José Moro del Secretario General de la Comisión sobre la continuidad de la exploración del NO. de África. $1 \mathrm{~h}$.

III. EXPEDICIÓN A CANARIAS DE CÉSAR LABRADO. 1905-1906

98 Conjunto documental sobre aspectos organizativos. $14 \mathrm{~h}$. Contiene:

- Nota de gastos

- 1905 junio 24, La Coruña-Pasaje a Tenerife y otros gastos (facturas)

- 1905, junio 1. St ${ }^{\text {a }}$ Cruz de Tenerife-facturas de hospedaje y papel.

- 1905, julio 8, 21, 27, 30. St St. St ${ }^{\mathrm{a}}$ Cruz de Tenerife-facturas de hospedaje, manutención.

- 1905, agosto 10, 12. La Palma-facturas de hospedaje

- 1905, septiembre 10. St ${ }^{\text {a }}$ Cruz de Tenerife-facturas de hospedaje.

- 1905, septiembre. Cádiz-facturas.

99 1905, noviembre 2, Santiago.

Carta de César Labrado a Ignacio Bolívar sobre una nota que envió con los gastos de la excursión a Canarias y acerca de las plantas de estas Islas. 2 h.

100 1906, enero 2, Cervera.

Carta de César Labrado a Ignacio Bolívar sobre su disposición a dosificar lo colectado en Canarias, comentándole que cuenta con libros adecuados en la Facultad de Farmacia de Santiago de Compostela. $2 \mathrm{~h}$.

101 Informe (borrador) sobre pesca y conservación de pescados con referencia a especial al banco canario-sahariano. $6 \mathrm{~h}$.

\section{ViAJE AL GOLFO DE NÁPOLES. [1905]}

102 Una visita a las regiones volcánicas del Golfo de Nápoles, por Ramón Llord y Gamboa. $40 \mathrm{~h}$. Se recoge la narración del viaje con todo tipo de datos geográficos y naturalistas. 


\section{LAS EXPEDICIONES CIENTÍFICAS ESPAÑOLAS EN LOS SIGLOS XIX Y XX}

V. COMISIÓN CIENTÍFICA A GALICIA. 1921-1929

103 1921, febrero 20.

Recorte del Diario «La voz de Galicia» en el que inserta una noticia de la Jnta de Ampliación de Estudios (JAE), en relación con el envío a Galicia de una Comisión Científica. $1 \mathrm{~h}$.

104 1922, octubre 10, Madrid.

Nota de Antonio Fernández a Ignacio Bolívar sobre gastos de personal y mantenimiento de la misión biológicas de Galicia. 1 h., (mec).

105 1922, noviembre 7, Santiago.

Carta de Cruz Gallastegui a Ignacio Bolívar sobre su carta del 30-X comentándole noticias de exámenes y otros asuntos... $1 \mathrm{~h}$.

106 1923, febrero 9, Santiago.

Carta de Cruz Gallastegui a Ignacio Bolívar sobre el envío que le hizo de una colmena y otros asuntos técnicos de su trabajo. $1 \mathrm{~h}$.

107 Anotaciones de cuentas. $1 \mathrm{~h}$.

108 1927, mayo 24, Torrevieja.

Carta sobre Nicolai Irnovich Vávilou a García Varela acerca de una conferencia del primero. $1 \mathrm{~h}$. (mec). Corre unida: nota sobre este asunto. $1 \mathrm{~h}$.

109 1927, junio 20, Madrid.

Carta (y copia) de I. Bolívar a Juan López Suarez acerca de que excursiones botánicas y zoológicas para el NO de España (Sierra de Ancares...) Galicia. Comenta composición del grupo expedicionario, gastos, material, etc. 4 h. (mec).

110 1927, junio 30, Santiago.

Carta de Ignacio Bolívar sobre el envío de unas muestras de «Evausita» y unas descripciones de yacimientos. $1 \mathrm{~h}$.

111 1927, julio 3, Celanova.

Carta a Ignacio Bolívar en la que el remitente comenta detalles de la excursión por Galicia y de las recolecciones realizadas hasta la fecha. $2 \mathrm{~h}$.

112 1927, julio 9, Madrid.

Carta a Ignacio Bolívar sobre la salida de Gómez Moreno para Galicia y la negativa del Ministerio de Estado para subvencionar a Río-Hortega y Sacristán. 1 h. (mec).

113 1927, julio 11, Madrid.

Carta de Antonio Fernández a Ignacio Bolívar sobre una postas mandada por Castillejo referentes a 2.000 pts., para la misión de Galicia. 1 h. (mec). 
114 1927, julio 30, Pontevedra.

Carta a Ignacio Bolívar poniéndose a disposición de los excursionista de Galicia y también que remitirá un ejemplar de «Evansita». $1 \mathrm{~h}$. (mec). Corre unida una nota del comité local de Pontevedra. 1 h. (mec).

115 1927, agosto 1, Madrid.

Carta de José Castillejo a Ignacio Bolívar sobre haber recibido del Sr. de la Sota un cheque de 2.500 pts. y de lo gastado en la misión de Galicia. 2 h. (mec).

116 1927, agosto 4, Pontevedra.

Carta de Luis Crespí a Ignacio Bolívar sobre su regreso de los Ancares y el del resto del grupo expedicionario. También comenta otros aspectos de esta excursión. $2 \mathrm{~h}$.

117 1927, agosto 4, Madrid.

Carta de José Castillejo a Luis Crespí sobre su recibí del cheque, cartas, cuentas y comprobantes de sus colectas. $1 \mathrm{~h}$. (mec).

118 1927, agosto 6, El Ventorrillo (Madrid).

Carta de Rioja a Ignacio Bolívar sobre los barcos y locales puestos a disposición del grupo expedicionario y los permisos para su actividad. $1 \mathrm{~h}$.

119 1927, agosto 8, Madrid.

Carta de José Castillejo a Ignacio Bolívar sobre la conveniencia de aplazar la excursión a Galicia. 1 h. (mec).

120 1927, agosto 10, Madrid.

Carta de José Castillejo a Ignacio Bolívar sobre autorización al personal científico del Museo para hacer excursiones. $1 \mathrm{~h}$. (mec).

121 1927, agosto 11, Madrid.

Carta de Rioja a Ignacio Bolívar sobre su traslado a la capital para hablar con él, con Lozano y Castillejo. $1 \mathrm{~h}$.

122 1927, agosto 13, Madrid

Carta de Luis Lozano a Ignacio Bolívar sobre las obras del Museo y sobre la campaña de septiembre y octubre. $1 \mathrm{~h}$. (mec).

112 31927, agosto 24, Pontevedra.

Informe de Luis Crespí a Ignacio Bolívar sobre la excursión para Galicia. Gastos y algunas aclaraciones al respecto. $2 \mathrm{~h}$.

124 1927, agosto 26, Celanova.

Carta a Ignacio Bolívar adjuntándole un talón de una caja para el Museo, con ratas e insectos... $1 \mathrm{~h}$. Corre unido: anotaciones de cuentas. $1 \mathrm{~h}$. 


\section{LAS EXPEDICIONES CIENTÍFICAS ESPAÑOLAS EN LOS SIGLOS XIX Y XX}

125 1927, agosto 27, El Ventorrillo (Madrid).

Carta de Rioja a Ignacio Bolívar sobre su visita a Madrid. Véase la carta de 11 de agosto. $1 \mathrm{~h}$.

126 1927, septiembre 13, Celanova.

Carta a Ignacio Bolívar sobre el envío de 2 frascos con ortópteros y de su salida para Santiago y la actividad docentes en esta ciudad. $2 \mathrm{~h}$.

127 [1927, diciembre].

Estado de cuentas de la excursión por Galicia. 2 h., (mec).

1281928.

«Comisión de Estudio en Galicia». Folletos impresos con la información de la J.A.E. sobre la Misión biológica de Galicia. 6 h.

129 1928, junio 11, Madrid.

Notificación de Ignacio Bolivar al Secretario de la Junta para Ampliación de Estudios con las cuentas de las «Misiones culturales de Galicia». 2 h. (mec). Corren unidas: notas fechadas en Madrid de 1928 con cuentas. $1 \mathrm{~h}$. (mec). Tres anotaciones sobre el mismo asunto. 4 h. «Ponencia de las Misiones culturales de Galicia». $1 \mathrm{~h}$.

1301928 , agosto 10. Ortigueira.

Carta de Federico Maciñeira a Ignacio Bolívar ofreciendo su cooperación con los enviados a Galicia.

131 1928, septiembre 18, Madrid.

Carta de Antonio Fernández a Ignacio Bolívar sobre la concesión de 2.500 pts de la Diputación de La Coruña para las misiones culturales de Galicia. 1 h.

132 1928, noviembre 19, Madrid.

Tarjeta postal de Terreiro a Ignacio Bolívar sobre unas cuartillas para corregir. $1 \mathrm{~h}$.

1331928 , noviembre 20.

Acuerdo de la Junta [de Ampliación de Estudios]. Sobre la composición de la Misión Biológica de Galicia. 1 h. (mec).

134 1929, febrero 15, Madrid.

Aviso a la Junta de Ampliación de Estudios de los costes de los encargados de la investigación arqueológica y preshistórica de Galicia. 2 h. mec.

135 1929, noviembre 9, Madrid.

Carta de Terreiro a Ignacio Bolívar sobre publicaciones de estudios gallegos... $2 \mathrm{~h}$. 


\section{ESTUDIOS Y ENVÍOS DE MATERIALES ZOOLÓGICOS DE GUINEA. 1924-1926}

136 1924, abril 28, Bata.

Carta de José Alonso a Ignacio Bolívar acerca de la necesidad que tiene de 40-50 1. de alcohol para poder enviar ejemplos para el Museo. $1 \mathrm{~h} ., \mathrm{f}$.

137 1924, octubre 20, Mikomeren (?).

Carta de José Alonso a José $\mathrm{M}^{\mathrm{a}}$ Benedito acerca de la gratificación que se le proporcione por parte del Museo para sus tareas naturales en la Colonia. También le comenta algo acerca de la prohibición en Guinea de la casa del chimpancé, gorila y orangután... 1 h., f.

138 1925, junio 4...

Carta de J. Benedito a Ignacio Bolívar sobre las cabezas de gorila, orangután y chimpancé que se ofrecen al Museo y el coste de la operación. También le habla del elefante que enviará. $1 \mathrm{~h}$.

139 1925, diciembre 4, Carabanchel [Madrid].

Presupuesto de José Alonso al director del Museo Nacional Ciencias Naturales sobre un grupo de animales de la Colonia de Guinea Ecuatorial (continente). 1 h., f., (mec).

140 1926, julio 21, Ebebergin (?).

Carta de José Alonso a Ignacio Bolívar. Sobre la remisión de mariposas, el craneo de un mandril, e insectos y de que se debe abonar su importe a su mujer a Madrid. También comenta noticias sobre otros animales... $1 \mathrm{~h}$., f. Corren unidos: carta desde la misma localidad de 30 de julio sobre otro envío de insectos. 1 h. Otra de 30 de septiembre sobre otro envío de 200 mariposas, 5 alacranes... 1 h., y otra de 26 de octubre sobre estos envíos y de otro de 950 mariposas y sobre sus problemas económicos... $1 \mathrm{~h}$.

141 Informe-diario dirigido al Gobernador General [de Guinea?] sobre la situación de los supervivientes de los poblados indígenas observados en ese territorio por el autor del escrito. Se dan noticias sobre enfermedades y condiciones higiénicas de sus habitantes. 15 h. (mec).

VII. [EXCURSIÓN DEL SR. LOZANO A RÍO DE ORO], 1933.

142 1933, julio 22, Cabo Jubi.

Carta de Luis Lozano a Ignacio Bolívar, informándole de su viaje, su paso por Canarias y noticias de la pesca en la costa del Sahara. Se incluye noticias de recolección garnisticos... $3 \mathrm{~h}$.

143 1933, agosto 2.

Carta de Lázaro a Ignacio Bolívar comentándole los pormenores del viaje, junto a su compañero Llorens y diciéndole que la colección de prod. naturales sigue aumentando incluyendo insectos, crustáceos, pieles de mamíferos etc... 


\section{LAS EXPEDICIONES CIENTÍFICAS ESPAÑOLAS EN LOS SIGLOS XIX Y XX}

144 1933, agosto 3, Villa Cisneros.

Carta de M. Llorens a Ignacio Bolívar comentándole noticias de sus recolecciones, de su próxima salida y de como pasan los días en esta zona. $1 \mathrm{~h}$.

145 1933, 30 agosto, la Agüera.

Carta de L. Lázaro a Ignacio Bolívar escrita a bordo del «Viera y Clavijo». Le comenta que ha quedado su estación en Villa Cisneros y de los especímenes que lleva, señalando detalles específicos de algunos de éstos. También le da noticias de sus próximos viajes hasta su regreso a Madrid. $2 \mathrm{~h}$.

146 1933, septiembre 15, Cabo Julio.

Carta de L. Lázaro a Ignacio Bolívar a bordo del «Lanzarote» caminos de las Canarias. Se informa de la desaparición de 2 insectos y del contingente de lo recolectado en el viaje. $2 \mathrm{~h}$.

VIII. ESTUDIOS Y EXPLORACIÓN FLORÍSTICA DE LA MANCHA DE GONZÁLEZ ALBO, 1934.

147 Estudio geobotánico y floristico de las Lagunas de Ruidera recogiendo las noticias de su viaje en 1933. Contiene, presupuestos y gastos de 2, excursiones realizadas por estas zonas. 4 h., f., (mec)

148 1934, julio 14, Ossa de Montiel.

Certificado de Valentín Alcocer a favor de José González Albo. 1 h., (mec).

149 1934, agosto 4.

Carta de José González Alboa a Ignacio Bolívar sobre el fin de su $2^{\mathrm{a}}$ excursión y de sus recolecciones y de haberle sido concedido por la Junta de Ampliación de Estudios la pensión para estudiar geografía Botánica en Francia... 2 h.

150 1934, noviembre 17, Montpellier.

Carta de González Albo a Ignacio Bolívar sobre haberle sido prolongado su misión. 1 h.

1511934.

«Generum plantarun ibericarun revisión critica» por José González Albo. Es un folleto (separata) de Cavanillesia con descripción del género Hutera.

\section{PROYECTO DE EXPEDICIÓN TRANSAFRICANA. 1933-36}

152 1933, diciembre 6, Barcelona.

Carta al director del Museo de Historia Natural acerca de la organización de una expedición a Africa Central, junto a otra que irá al Amazonas. Comenta la adquisición para ello de un velero y los propósitos de la empresa. 1 h., f., (mec.) 
1531935

Folleto impreso «Expedición Española Científica Transafricana». 16 h. + tapas.

154 1936, febrero 28, Barcelona.

Carta de Joaquín Poch y José Rovira Ignacio Bolívar sobre gastos de la Expedición Transafricana que tienen prevista su salida a principios de octubre. 1 h., f., (mc).

155 1936, febrero 29. Barcelona.

Carta de Tomás Martín, Manuel López, Joaquín Poch y José Rovira a Ignacio Bolívar solicitando apoyo a su Expedición Transafricana y sobre su composición. 1 h., f., (mec). Corre unida, relación con los miembros del Patronato y de la propia Expedición. 1 h., (mec).

156 1936, marzo 23, Barcelona.

Carta de José Rovira Petit a Ignacio Bolívar, mostrando su sorpresa para que la Expedición al Amazona se dirigirá hacia Africa, donde se dirigirá la suya. Propone una colaboración entre ambas... 1 h., f., (mec).

157 1936, marzo 29, Barcelona.

Carta de José Rovira a Ignacio Bolívar sobre su previsión de explorar las colonias del Golfo de Guinea. Solicita algunos ejemplares del Proyecto de Expedición Transafricana. 1 h., f., (mec).

158 1936, mayo 9, Madrid

Carta de Ignacio Bolívar al Presidente del Patronato de la Expedición Transafricana donde se apoyo a la Expedición y aceptando el ofrecimiento de José Cuatrecasas para formar parte de la Expedición 1 h., (mec).

\section{COMISIÓN CIENTÍFICA DE IFNI. 1834-35}

159 1934, abril 27, Madrid.

Circulares (varios duplicados), dirigidas a Eduardo Hernández Pacheco con los nombramientos de los miembros de la Comisión y las condiciones en que se desarrollará ésta. 11 h., (mec.) $+6 \mathrm{f}$.

160 1934, abril 29, Madrid.

Carta de Luis Lozano a Eduardo Hernández Pacheco acerca de una reseña sobre la «Comisión Científica a Ifni» aparecida en el periódico «Ahora». 2 h., (mec). Corren unidos: recortes del periódico y telegrama con diversas preguntas sobre la Comisión. 2 h., (mec).

1611934.

Facturas y recibos con cargo a la Comisión Científica de Ifni. 111 h., (mec). Contiene duplicados y copias, unas manuscritas y otras mecanografiadas en tamaño folio, cuartilla, etc. 


\section{LAS EXPEDICIONES CIENTÍFICAS ESPAÑOLAS EN LOS SIGLOS XIX Y XX}

162 1934, mayo 3, Madrid.

Carta de Miguel Santaló a Eduardo Hernández Pacheco reiterándole su deseo de ir como «Agregado» a la Comisión de Ifni. 1 h. Corre unida: contestación de 6 de mayo. 1 h., (mec).

163 1934, mayo 7, Madrid.

Circulares del Director General de la Presidencia del Consejo de Ministros para que se ponga a disposición de Eduardo Hernández Pacheco diversos asuntos para la Comisión. 2 h., f., (mec.)

164 1934, mayo 10, Madrid.

Carta de Eduardo Hernández Pacheco al Ministro de la Guerra pidiéndole material para la Comisión... 1 h., (mec). Corren unidos: Comunicaciones del Intendente $14 \mathrm{de}$ mayo a Eduardo Hernández Pacheco para la que se facilita diverso material. $1 \mathrm{~h}$., (mec). Otra del 16 de mayo sobre la remesa a las Palmas de 5 tiendas de cajones. $1 \mathrm{~h}$., mec. Otra de 18 de mayo dirigida a Manuel Seco sobre ese material. 1 h., mec. Otra de la misma fecha ordenando remitir al «Pabellón Docker» los materiales. 1 h., (mec).

165 1934, mayo 10, Madrid.

Propuesta de Eduardo Hernández Pacheco de Carlos Crespi Jaume como Topógrafo de la Comisión. 1 h., (mec). Acompaña con fecha 18 de mayo, la circular de este nombramiento. 1 h., f., (mec)

166 1934, mayo 14, 16 y 18. Madrid.

Cartas y comunicaciones sobre material de campaña. Véase $n^{\circ} 164$.

167 1934, mayo 24, Madrid.

Carta a Eduardo Hernández Pacheco sobre los problemas de salir hacia Africa desde Cádiz. 1 h., (mec).

168 Cádiz.

Telegrama del inspector Provincial de Sanidad al Inspector General comunicando la salida de 14 bultos. 1 h., (mec). Acompaña notificación de 28 de mayo de Eduardo Hernández Pacheco sobre los 14 bultos que se han de facturar. 1 h., (mec).

169 1934, mayo 26. Madrid.

Comunicación a Eduardo Hernández Pacheco sobre la concesión de una gratificación. Se especifican los contenidos. 1 h., f. (mec.)

170 1934, mayo 28, Madrid.

Notificación de salida. Véase $n^{\circ} 168$

171 1934, mayo 30, Madrid.

Recibos de Eduardo Hernández Pacheco y de Carlos Crespi, de diversas cantidades anticipadas para gastos de la Comisión. 5 h., (mec). Varias de ellas rotas. 


\section{J. LUIS MALDONADO POLO}

172 [1934].

Dos folletos de las Líneas Aéreas Postales Españolas, con el extracto de las condiciones generales de transporte de viajeros y equipajes. (Impresas).

173 1934, mayo 31, Madrid.

Notificación a Leandro Pita y Romero sobre copias de cartas que le dirige José Mosqueira sobre cuestiones pesqueras. $1 \mathrm{~h}$., (mec).

174 1934, junio 2.

Copia de telegrama del Subsecretario Presidencial Consejo Ministros al Gobernador Político-Militar de Ifni. Sobre la salida de 3 miembros de la Comisión y los 6 restantes embarcarán el día 4 en Cádiz para Las Palmas... . 1 h., (mec).

175 1934, 2, Madrid.

Comunicación de Eduardo Hernández Pacheco al Subsecretario de la Marina Civil sobre que Luis Lozano Rey ha sido nombrado zoólogo de la Comisión. 1 h., (mec).

176 Comunicación a la Junta para Ampliación de Estudios que tanto el comunicante como Eduardo Hernández Pacheco tienen por misión estudiar la geografía y geología de Ifni respectivamente... . $1 \mathrm{~h}$., (mec).

177 1934, junio 2, Madrid.

Relación de gastos de material de la Comisión de Ifni, por Eduardo Hernández Pacheco. 2 h., (mec).

178 1934, junio 10. Ifni.

Saludo de Eduardo Hernández Pacheco al Gobernador de la provincia y telegrama para el comandante. Dato sobre víveres y menaje de cocina... $1 \mathrm{~h}$., (mec). Corre unido comunicación de Benigno González-Alles, fechado, junio 11, Ifni, acusando recibo del escrito anterior y propone hacer un levantamiento hidrográfico de Ifni. 1 h., (mec).

179 1934, junio 13, Sidi Ifni.

Comunicación de Carlos Pedemonte a Eduardo Hernández Pacheco sobre la ayuda de algún marino a Luis Lozano en las tareas de pesca... . 1 h., (mec).

180 1934, junio 14, Sidi Ifni.

Comunicación de Eduardo Hernández Pacheco sobre la necesidad de vacunación «antipestosa» del personal de la Comisión. 1 h., (mec).

181 1934, junio 20, Sidi Ifni.

Comunicación de Carlos Pedemonte a Eduardo Hernández Pacheco de un telegrama sobre el envío de un «chinchorro» y del importe total 1 h., (mec). 


\section{LAS EXPEDICIONES CIENTÍFICAS ESPAÑOLAS EN LOS SIGLOS XIX Y XX}

182 1934, junio 27, Puerto de la Luz.

Radiotelegrama dirigido a Eduardo Hernández Pacheco sobre la salida en el vapor «Consuelo Humidobro» de víveres y utensilios para la Comisión. 1 h., (en sobre original). Corre unida comunicación de 28 de junio de Carlos Pedemonte a Eduardo Hernández Pacheco de lo anterior. 1 h., (mec).

183 1934, junio 30, Ifni.

Relación de víveres que la Intendencia de Ceuta proporcionó a la Comisión. 2 h. Acompaña: notas y vales sobre víveres y comidas. $16 \mathrm{~h}$.

184 1834, julio 2, Sidi Ifni.

Comunicación de Carlos Pedemonte a Eduardo Hernández Pacheco sobre la remisión de las Palmas de 50 cartuchos. 1 h., (mec).

185 1934, julio 2, La Agüera.

Carta a Eduardo Hernández Pacheco sobre detalles de la zona africana donde irá la Comisión. 2 h., f., (mec).

186 1934, julio 3, Sidi Ifni.

Sobre el abono a la casa comercial «Pedro Fluiters» de $1.006^{`} 15$ pts. 2 h.

187 1934, julio 5, Sidi Ifni.

Trasmisión de Carlos Pedemonte a Eduardo Hernández Pacheco de un telegrama sobre incumplimiento de sus ordenes de no rebasar los comisionados los puertos franceses. 1 h., (mec). Acompañan minutas de Eduardo Hernández Pacheco, de 8 de julio, explicando esta circunstancia. $2 \mathrm{~h}$.

188 1934, julio 6, Agadir.

Carta de F. Escalera sobre su viaje hasta esta localidad y los pormenores de éste tránsito. 1 h., f., a lápiz.

189 1934, julio 8, Sidi Ifni.

Comunicación a Eduardo Hernández Pacheco sobre que acompaña a tres tenientes a revisar armamento y ponerlo para reconocer el territorio. $1 \mathrm{~h}$.

190 1934, julio 12, Sidi Ifni.

Telegrama a Eduardo Hernández Pacheco del Gobernador sobe que la Comisión puede reconocer Uggu. 1 h., (mec).

191 1934, julio 13, Sidi Ifni.

Carta de Carlos Pedemonte a Eduardo Hernández Pacheco despidiéndose de él para tomar posesión en Tetuán de su nuevo cargo. $1 \mathrm{~h}$. 
1921934 [Julio].

Relaciones de gastos (personal, material, etc) de la Comisión Científica... . 14 h., (mec).

193 [Junio, 1934].

Relación de «Cantidades satisfecha a Luis Lozano». Viaje de Ifni.

$6 \mathrm{H}$. , (mec).

194 1934, julio 19. Sidi Ifni.

Recibo del «Depósito de Intendencia» de varios efectos de la Comisión. 1 h.

195 1934, julio. Sidi Ifni.

Facturas y vales del Deposito de Intendencia de Ifni sobre varias cantidades de alimentos para la Comisión Científica. 24 h.

196 1934, julio 24, 26, y 27.

Talones sobre equipajes de la Comisión. 3 h.

197 1934, julio 29, Sidi Ifni

Notificación de Francisco Sanz sobre remisión de tres cartas para los Comisionados. 1 h.

198 1934, julio 30.

Relación sobre pertrechos y equipos de campo. 1 h., f., a lápiz.

199 1934, agosto 2, Madrid.

Informe del Ingeniero geógrafo sobre la conveniente cartografía de Ifni. 16 h., f., (mec).

200 1934, agosto 4, Madrid.

Carta del Presidente de la Comisión al Director General de Sanidad y al habilitado de la Presidencia del Consejo de Ministro, sobre el abono a la Compañía de Ferrocarriles M.Z.A. la cantidad de $1.065^{\prime} 50$ pts. 2 h., (mec).

201 1934, agosto 5, Madrid.

Cartas a Benigno Martínez Portillo y a Carlos Pedemonte agradeciendo las atenciones prestadas a los miembros de la Comisión. 2 h., a lápiz.

202 1934, agosto 6, Madrid.

Carta a José González Deleito agradeciendo las atenciones prestadas a los miembros de la Comisión. 1 h. a lápiz.

203 1934, agosto 8, Madrid.

Carta de Arturo Revoltós a Eduardo Hernández Pacheco sobre los gastos que ha hecho en Ifni y los pases de su trabajo. 2 h., (mec).

204 1934, agosto 10, Madrid.

Carta de Carlos Crespí y Caballero a Hernández Pacheco sobre gastos de la Comisión. 2 h. 


\section{LAS EXPEDICIONES CIENTÍFICAS ESPAÑOLAS EN LOS SIGLOS XIX Y XX}

205 1934, agosto 14, Madrid.

Cuentas de gastos de los 25.000 pts. anticipadas a Hernández Pacheco para material. 12 h., + 11 f., (mec).

206 1934, agosto 14, Melilla.

Carta de Luis Lozano a Hernández Pacheco, informándole sus sinsabores respecto a la Comisión y sobre liquidación de gastos de ella. Se incluye una relación de estos. 3 h., + 1 h., f., (mec).

207 1934, agosto.

Cómputo de carta, sobre telegramas... de Luis Bellón a Hernández Pacheco, Lozano, etc. Sobre el regreso de la Comisión y otros aspectos de ésta... .17 h., 2 f., (mec).

208 1934, agosto 20, Tánger.

Carta de F. Escalera a Eduardo Pacheco sobre la relación de gastos efectuados por él en Ifni. 1 h., f.

209 1934, agosto 23, Madrid.

Notificación al Presidente de la Comisión Científica de Ifni sobre la habilitación del aeródromo de Ifni. 1 h., mec.

210 1934, octubre 1.

Sobre el pago a la Compañía de los FF.CC. Andaluces de 258`19 pts, para gastos de transporte de la Expedición. 1 h., (mec).

211 1934, octubre 15, Madrid.

Petición de Eduardo Hernández Pacheco al Secretario de Marruecos de 50.000 pts. para la Comisión. 1 h., (mec). Acompaña carta de 21 de noviembre de Guillermo Moreno sobre este asunto. 1 h., f., (mec).

2121934 , octubre 18.

Carta de Eduardo Roch sobre la carta geológica de Marruecos... (en francés). 1 h.

213 1934, octubre 29, Madrid.

Comunicación de Eduardo Hernández Pacheco al Secretario Técnico de Marruecos comunicándole el envío de las cuentas de dietas y gastos del viaje. 1 h., (mec).

214 1934, noviembre 10, Madrid.

Carta de Eduardo Roch sobre un esquema de la carta geológica de Ifni. 1 h., (mec) Acompaña con fecha de 14 de noviembre una carta de Eduardo Roch sobre geología. 1 h., f., (mec). Otra de 20 de noviembre en contestación a ésta. 2 h., (mec).

215 1934, noviembre 13, Madrid.

Notificación de Ramón a Eduardo Hernández Pacheco sobre dos relaciones de fichas. 1 h., f., (mec). 
216 1934, noviembre 24, Madrid.

Carta del Secretario Técnico de Marruecos a Eduardo Hernández Pacheco sobre la remisión de justificación de cuentas. 1 h., (mec)., f.

217 1934, noviembre 30, Madrid.

Carta de Eduardo Hernández Pacheco al Secretario de la Presidencia de Gobierno sobre que Escalera irá a Ifni en diciembre y nombra a Leopoldo Calvo veterinario de la Expedición. 1 h., (mec). Corre unida con la misma fecha una carta del Subsecretario a Eduardo Hernández Pacheco trasmitiéndole este asunto. 1 h., f., (mec).

218 1934, diciembre 8

Relación de cuentas de la Comisión realizada por Eduardo Hernández Pacheco. 2 h., f.

219 1934, diciembre 10, Rabat.

Carta de Eduardo Roch sobre la geología de Ifni, (en francés). 1 h., f., (mec).

220 1934, diciembre 11, Madrid.

Carta duplicada de Eduardo Hernández Pacheco al Delegado Gubernativo de Ifni que en esta fecha se dá orden de portada para Ifni. 2 h., (mec).

221 1934, diciembre 19, Madrid.

Nota de Gerardo Sala Gómez a Eduardo Hernández Pacheco en la que le adjunta facturas de los trabajos de la película. $1 \mathrm{~h}$.

222 1934, diciembre 21, Madrid.

Carta de Eduardo Hernández Pacheco sobre una copia del informe que envío al Gobernador sobre la exp. a Ifni y de una fotografía. 2 h., a lápiz.

223 1934, diciembre.

Nota de Luis Lozano sobre material fotográfico. $1 \mathrm{~h}$.

224 [1934].

Informe de Eduardo Hernández Pacheco de las actividades de la Expedición a Ifni y que al final de la misma se repongan los gastos adelantados por los expedicionarios. 1 h., (mec).

225 [1934, diciembre].

«Declaración jurada» hecha por F. M. de la Escalera de gastos de la Comisión. 2 h., f., (mec).

226 [1934, informe descriptivo] del reconocimiento del NO de África, con alusión a Canarias. 7 h., a lápiz.

227 [1934 á 1935].

Anotaciones a lápiz sobre rocas de Ifni. 1 h., f. 


\section{LAS EXPEDICIONES CIENTÍFICAS ESPAÑOLAS EN LOS SIGLOS XIX Y XX}

228 1935, enero 17.

Carta de Antonio Morales a Eduardo Hernández Pacheco sobre la proyectada expedición al Sahara. $1 \mathrm{~h}$.

229 1935, mayo 18, [Barcelona].

Carta de M. San Miguel a Eduardo Hernández Pacheco comentándole pormenores de la Comisión a Ifni. 1 h., (mec).

230 1935, junio 26, Las Palmas.

Carta a Eduardo Hernández Pacheco sobre el reconocimiento del NO de Africa. 2 h.

231 1935, diciembre 23, Barcelona.

Carta de Bataller a Eduardo Hernández Pacheco sobre análisis geológicos en Africa, ¿tal vez fosfatos?. $2 \mathrm{~h}$.

232 [1935].

Memoria de Eduardo Hernández Pacheco sobre la Expedición Científica a Ifni. 58 h., a lápiz.

233 [1934-1935].

- Rótulos de la Expedición Científica a Ifni, por Eduardo Hernández Pacheco. 25 h.

- Anotación en tarjeta sobre el «Libro del Sahara español». 1 h.

- Notas bibliográficas sobre Ifni-Sahara. 12 h. (a lápiz)

XI. «EXPEDICIÓN IGLESIAS AL AMAZONAS». 1936

234 1936, enero 31. Madrid.

Oficio del Subsecretario al director del MNCN, comunicándole que Federico Bonet Marco ha terminado los trabajos de organización de la Expedición 1 h., (mec).

235 1936, octubre 13, Madrid.

Disposiciones (original más copia) del Ministerio de Instrucción Pública sobre el destino del material que estaba dispuesto para la Exp. al Amazonas. 4 h., (mec).

236 1936, noviembre 3, Madrid.

Oficio del Director provincial del MNCN al Ministro de Instrucción pública, presentándole un inventario de la «primera entrega» del material destinado para la Expedición del Amazonas. Se adjunta dicho inventario fechado en Madrid, el 30 de octubre y firmado por Antonio Zulueta. 10 h., (mec)., f.

XII. CAMPAÑAS ICTIOLÓGICAS Y PESQUERAS. 1939

237 [1939]

Proyecto de Campaña Ictiológica en Palma de Mallorca, Málaga, Melilla, Tánger, Ceuta, Islas Columbretes y Alborán. Se recogen las labores a realizar, finalidad de las mismas, miembros integrantes, material y presuestos. $3 \mathrm{~h} .$, f. (mec.) 
238 1939, diciembre 29, Madrid

Proyecto de la segunda Campaña científico-pesquera a las costas del Sahara español, por Luis Lozano y Rey. 4 h. (mec)

\section{Trabajos PReParatorios de la expedición a Guinea, SAHARA E IfNi. 1939-40}

239 1939, noviembre 8, Madrid.

Carta del director del MNCN, solicitando apoyo para un plan de expediciones a Guinea Continental, Fernando Poó, Rio de Oro e Ifni, Sahara y su costa. 2 h., (mec).

240 1939, noviembre 9, Madrid.

«Proyecto de expediciones científicas» a los territorios señalados. 2 h., f., (mec).

241 1940, Mayo 25, Madrid.

Nota sobre 7 kg. de arsénico y 30 de alambre para la Expedición de Guinea. 1 h.

242 1940, julio 13, Madrid.

Informe del director de la Expedición. que contiene los trabajos y estudios preparatorios sobre la Expedición a Guinea. 7 h., + 1 mapa, (mec).

243 1940, agosto 15, Bata.

Carta a Pedro Novo, sobre la marcha de la campaña, nombramiento, sustituciones... 1 h., (mec).

244 1940, agosto 15, Bata.

Nota de Llorens sobre su permanencia o regreso de Africa. $1 \mathrm{~h}$.

245 1940, septiembre 11, Madrid.

Dos cartas de Pedro Novo a Manuel Plaza y a Lizaur, Gil Lltyget, Llorens y Curaus. Comunicando al primero que le remita la carta que le enviaron los demás a los que también les comunica su decisión. 2 h., (mec).

246 1940, octubre 10.

Cablegrama sobre la incorporación de Llorens. 1 h., (mec).

\section{EXPEDICION A FILIPINAS. S.F.}

247 Relato de Domingo Sánchez, pp. 22-36 y 32-118. Se agrega una parte de la excursión por Samar verificada por Regino García, ayudante botánico y por Domingo Sánchez, auxiliar zoólogo. Relatada por éste último, en la que se recogen descripciones y noticias de especies zoológicas. $3 \mathrm{~h}$. 